Pacific Journal of Mathematic 


\section{ON A RADON-NIKODYM THEOREM FOR FINITELY ADDITIVE SET FUNCTIONS}

\section{R. B. Darst and Euline Green}

The purpose of this note is to comment on and extend recent results of $C$. Fefferman. A proof of his Radon-Nikodym theorem that is, perhaps, more amenable to generalization is given. A Lebesgue decomposition is also obtained.

Since the notations in [3] and [7] conflict, we shall make the following compromises in notation and terminology, and beg the reader's indulgence.

Let $S$ be a set, $\Sigma$ be an algebra of subsets of $S, C$ be the complex numbers, and $R$ be the real numbers. Let $H(C)=H(S, \Sigma: C)$ denote the set of all bounded, complex valued, finitely additive set functions on $\Sigma$. Then $H(R)$ will denote the real valued elements of $H(C)$. If $\alpha \in H(C)$ and $E \in \Sigma$, we denote the total variation of $\alpha$ over $E$ by $v(\alpha, E)$. If $\alpha, \beta \in H(C)$ then

(i ) $\alpha$ is absolutely continuous with respect to $\beta(\alpha \ll \beta)$ means: given $\varepsilon>0$, there exists $\delta>0$ such that $v(\beta, E)<\delta(E \in \Sigma)$ implies $v(\alpha, E)<\varepsilon$.

(ii) $\alpha$ is singular with respect to $\beta(\alpha \perp \beta)$ means: given $\varepsilon>0$, there exists $E \in \Sigma$ such that $v(\alpha, E)<\varepsilon$ and $v(\beta, S-E)<\varepsilon$.

The classical Radon-Nikodym theorem (eg., [6, Th. III. 10.2]) asserts that if $\Sigma$ is a sigma algebra and $\lambda$ is a countably additive element of $H(C)$, then $\lambda$ can be given an integral representation with respect to a nonnegative, countably additive element $\mu$ of $H(R)$ if, and only if, $\lambda$ is absolutely continuous with respect to $\mu$.

In 1939, S. Bochner published a generalization ([1]) which removed the restrictions that $\Sigma$ be a sigma algebra and that the set functions be countably additive. Then S. Bochner and R. S. Phillips [2] used a vector lattice approach to give a new proof of Bochner's Theorem and, also, to obtain a Lebesgue decomposition. S. Leader [8] studied the $L^{p}$-spaces associated with finitely additive measures. A representation for the case where $\mu \in H(R)$ appeared ([3]) in 1962. Theorem III. 10.7 of [6] supplements the classical theorem by allowing $\mu$ to be complex valued, and recently C. Fefferman ([7]) extended the latter result to the case of a general algebra of subsets of a set.

Let us turn to some comments on the paper of Fefferman.

(i) The definition of absolute continuity given in [7] seems to contain a misprint: Suppose that $S=[-1,1], \Sigma$ is the sigma algebra of Lebesgue measurable subsets of $S, \alpha$ is Lebesgue measure $m$ res- 
tricted to $[-1,0]$ (i.e., $\alpha(E)=m(E \cap[-1,0])), \beta$ is Lebesgue measure restricted to $[0,1], \mu=\alpha-\beta, \gamma=\alpha+\beta$ and $E=S$. Then $\mu(E)=0$ while $\gamma(E)=2$ and, hence, $\gamma$ is not absolutely continuous with respect to $\mu$, according to [7]. Replacing $|\mu(E)|$ by $v(\mu, E)$ or by sup $\{|\mu(F)|$; $F \in \Sigma, F \subset E\}$ in the definition of absolute continuity given in [7] prevents the pathology illustrated by the preceding example.

(ii) After correcting the misprint, one should replace the last statement in [7, p. 35, paragraph 1$]$ by "Unless $\gamma$ is bounded and countably additive the last two definitions need not be equivalent." (See [4, Th. 3.4]. For a counterexample to the original statement, let $S$ be the positive integers, let $\Sigma$ be the $\sigma$-algebra of all subsets of $S$, let $\mu(E)=\Sigma_{n \in E} 2^{-n}$, and let $\gamma$ be a Banach measure).

(iii) Existence of a Lebesgue decomposition in the setting of [7] follows immediately from [5] upon letting $T_{\varepsilon}=\{E \in \Sigma ; v(\mu, E)<\varepsilon\}$.

(iv) The first four lemmas in [7] are obtained in [3] by a technical modification of a standard proof of the classical Radon-Nikodym theorem.

(v) While the last two lemmas in [7] are neat, our argument seems to make certain generalizations more transparent. Consider, for example, finitely additive set functions on $\Sigma$ to a Banach space over $R$ with basis. The coordinate functionals are finitely additive, and if enough conditions are imposed, then our procedure can be applied.

For the sake of completeness and clarity, we state Theorem 2.2 of [3].

If $f, g \in H(R)$, then there exist uniquely functions $h$ and $s$ in $H(R)$ such that

(i) $h \ll g$,

(ii) $s \perp g$,

(iii) $f=h+s$

(iv) $E \in \Sigma$ implies $v(f, E)=v(h, E)+v(s, E)$.

Moreover, there exists a sequence $\left\{y_{n}\right\}$ of $(S, \Sigma: R)$-simple functions which converges in $g$-measure and such that if $h_{n}(E)=\int_{E} y_{n} d g$ for each $E \in \Sigma$, then $\lim _{n} v\left(h-h_{n}, S\right)=0$.

The following is the desired extension.

THEOREM 1. Suppose $\alpha, \beta \in H(C)$ and $\alpha \ll \beta$. Then there exists a sequence $\left\{z_{n}\right\}$ of $(S, \Sigma: C)$-simple functions such that if $\alpha_{n}(E)=$ $\int_{E} z_{n} d \beta$ for each $E \in \Sigma$, then $\lim _{n} v\left(\alpha-\alpha_{n}, S\right)=0$.

In order to apply Theorem 2.2 , we need the following 
LEMma 1. Suppose

(i) $\alpha, \beta, \gamma \in H(C)$,

(ii) $\left\{g_{n}\right\}$ is a sequence of (S, $\left.\Sigma: C\right)$-simple functions such that, if $\gamma_{n}(E)=\int_{E} g_{n} d \alpha$ for each $E \in \Sigma$, then $\lim _{n} v\left(\gamma-\gamma_{n}, S\right)=0$,

(iii) $\beta \perp \gamma$.

Then there exists a sequence $\left\{h_{n}\right\}$ of $(S, \Sigma: C)$-simple functions such that if $\gamma_{n}^{\prime}(E)=\int_{E} h_{n} d \alpha$ and $\gamma_{n}^{\prime \prime}(E)=\int_{E} h_{n} d(\alpha+\beta)$ for each $E \in \Sigma$, then

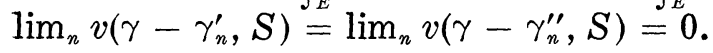

Proof. Let $M_{n}=\max \left\{\left|g_{n}(x)\right| x \in S\right\}$. Since $\gamma \perp \beta$, we choose a sequence $\left\{A_{n}\right\}$ of elements of $\Sigma$ such that $v\left(\beta, A_{n}\right)<a_{n}$ and $v\left(\gamma, S-A_{n}\right)<a_{n}$ where $a_{n}=\min \left\{1 / n M_{n}, 1 / n\right\}$. Define $\left\{h_{n}\right\}$ by $h_{n}(x)$ $=g_{n}(x)$ if $x \in A_{n}$ and $h_{n}(x)=0$ if $x \in S-A_{n}$. Let $\varepsilon>0$ be given and choose $N$ so large that $n \geqq N$ implies both $v\left(\gamma-\gamma_{n}, S\right)<\varepsilon / 3$ and $1 / n<\varepsilon / 3$. Then if $E \in \Sigma$ and

$$
\begin{aligned}
n \geq & N,\left|\gamma(E)-\gamma_{n}^{\prime}(E)\right| \leqq\left|\gamma(E)-\gamma_{n}(E)\right|+\mid \gamma_{n}(E)-\gamma_{n}^{\prime}(E) \\
& -\gamma\left(E \cap\left(S-A_{n}\right)\right)|+| \gamma\left(E \cap\left(S-A_{n}\right)|<(\varepsilon / 3)+| \gamma_{n}\left(E \cap\left(S-A_{n}\right)\right)\right. \\
& -\gamma\left(E \cap\left(S-A_{n}\right)\right) \mid+v\left(\gamma, S-A_{n}\right)<(\varepsilon / 3)+(\varepsilon / 3)+(\varepsilon / 3)=\varepsilon .
\end{aligned}
$$

Therefore, $\lim _{n} v\left(\gamma-\gamma_{n}^{\prime}, S\right)=0$. Finally, for $E \in \Sigma$ and

$$
\begin{aligned}
n \geq & N,\left|\gamma(E)-\gamma_{n}^{\prime \prime}(E)\right| \leqq\left|\gamma(E)-\gamma_{n}^{\prime}(E)\right|+\left|\int_{E} h_{n} d \beta\right|<\varepsilon \\
& +\left|\int_{E \cap A_{n}} g_{n} d \beta\right| \leqq \varepsilon+M_{n} V\left(\beta, A_{n}\right) \\
& <\varepsilon+M_{n} \frac{1}{n M_{n}}<\varepsilon+\frac{\varepsilon}{3}<2 \varepsilon .
\end{aligned}
$$

Therefore $\lim _{n} v\left(\gamma-\gamma_{n}^{\prime \prime}, S\right)=0$, and Lemma 1 is proved.

Proof of Theorem 1. Any $\alpha \in H(C)$ may be separated into its real and complex parts, so it suffices to prove the theorem when $\alpha$ is real valued. According to Theorem 2.2, we can express $\beta$ as follows: $\beta=\mu+i \nu=\left(\mu_{1}+\mu_{2}\right)+i\left(\nu_{1}+\nu_{2}\right)$ where $\mu, \nu, \mu_{1}, \mu_{2}, \nu_{1}, \nu_{2} \in H(R)$ and
(a) $\mu_{1} \perp \nu$
(b) $\mu_{2} \ll \nu$
(c) $\nu_{1} \perp \mu$
(d) $\nu_{2} \ll \mu$.

We also write $\alpha=\alpha_{1}+\alpha_{2}$ where
(a) $\alpha_{1} \perp \nu$
(b) $\alpha_{2} \ll \nu$.

Notice now that $\alpha_{1} \ll \mu_{1}$. The reason for this is: 
$\alpha_{1} \ll \alpha \ll \beta$ and (2) (a) imply $\alpha_{1} \ll \mu=\mu_{1}+\mu_{2}$. But (1) (b) and (2) (a) imply $\alpha_{1} \perp \mu_{2}$, and hence $\alpha_{1} \ll \mu_{1}$. Therefore, again by Theorem 2.2, there exists a sequence $\left\{f_{n}\right\}$ of $(S, \Sigma: R)$-simple functions such that $\lim _{n} \int_{E} f_{n} d \mu_{1}=\alpha_{1}(E)$ uniformly for $E \in \Sigma$. Since $\alpha_{1} \perp \nu$ and $\alpha_{1} \perp \mu_{2}$, we conclude that $\alpha_{1} \perp \mu_{2}+i \nu$, and apply Lemma 1 to get a sequence $\left\{f_{n}^{\prime}\right\}$ of $(S, \Sigma: C)$-simple functions such that

$$
\begin{aligned}
& \lim _{n} \int_{E} f_{n}^{\prime} d\left(\mu_{1}+\left(\mu_{2}+i v\right)\right)=\lim _{n} \int_{E} f_{n}^{\prime} d \beta=\alpha_{1}(E) \\
& \text { uniformly for } E \in \Sigma .
\end{aligned}
$$

Now we look at $\alpha_{2}$, and write it as $\alpha_{2}=\alpha_{2}^{\prime}+\alpha_{2}^{\prime \prime}$ where

$$
\text { (a) } \alpha_{2}^{\prime} \perp \mu
$$

(b) $\alpha_{2}^{\prime \prime} \ll \mu$.

As before, $\alpha_{2}^{\prime} \ll \alpha_{2} \ll \nu$, thus (3) (a), (1) (d) imply $\alpha_{2}^{\prime} \perp \nu_{2}$, and hence $\alpha_{2}^{\prime} \ll \nu_{1}$. Also $\alpha_{2}^{\prime} \perp \nu_{2}$ and $\alpha_{2}^{\prime} \perp \mu$ imply $\alpha_{2}^{\prime} \perp \nu_{2}-i \mu$. Therefore by Theorem 2.2 and Lemma 1 , there exists a sequence $\left\{g_{n}^{\prime}\right\}$ of $(S, \Sigma: C)$ simple functions such that

$$
\lim _{n} \int_{E} g_{n}^{\prime} d\left(\nu_{1}+\left(\nu_{2}-i \mu\right)\right)=\lim _{n} \int_{E}(-i) g_{2}^{\prime} d \beta=\alpha_{2}^{\prime}(E)
$$

uniformly for $E \in \Sigma$.

We are still left with $\alpha_{2}^{\prime \prime}$. But (3) (b), $\alpha_{2}^{\prime \prime} \ll \alpha_{2}$, (2) (b), and (1) (a) imply $\alpha_{2}^{\prime \prime} \perp \mu_{1}$, hence $\alpha_{2}^{\prime \prime} \ll \mu_{2}$. Hence there exists a sequence $\left\{k_{n}\right\}$ of $(S, \Sigma: R)$-simple functions such that $\lim _{n} \int_{E} g_{n} d \mu_{2}=\alpha_{2}^{\prime \prime}(E)$ uniformly for $E \in \Sigma$. But we cannot apply Lemma 1 here since we do not have $\alpha_{2}^{\prime \prime} \perp \mu_{1}+i \nu$. However, (1) (d), $\nu_{2} \ll \nu$, and (1) (a) imply $\nu_{2} \ll \mu_{2}$. Therefore there exists a sequence $\left\{l_{n}\right\}$ of $(S, \Sigma: R)$-simple functions such that $\lim _{n} \int_{E} l_{n} d \mu_{2}=\nu_{2}(E)$ uniformly for $E \in \Sigma$. Let $K_{n}=\max$ $\left\{\left|k_{n}(x)\right|, x \in X\right\}$, and, by taking a subsequence of $\left\{l_{n}\right\}$ if necessary, suppose $v\left(\int l_{n} d \mu_{2}-\nu_{2}, E\right)<1 / n K_{n}$ for all $E \in \Sigma$. Consider the sequence $\left\{h_{n}\right\}$ where $h_{n}=k_{n} /\left(1+i l_{n}\right)$. Notice that $h_{n}$ is a step function, is defined everwhere since $1+i l_{n}$ is never zero, and $\left|h_{n}(x)\right| \leqq\left|k_{n}(x)\right|$ for each $x \in S$. Let $\varepsilon>0$, and choose $N$ so large that $n \geqq N$ implies both

(i) $\left|\alpha_{2}^{\prime \prime}(E)-\int_{E} k_{n} d \mu_{2}\right|<\varepsilon / 2$ for all $E \in \Sigma$ and

(ii) $1 / n<\varepsilon / 2$.

If $E \in \Sigma$, then $\mid \alpha_{2}^{\prime \prime}(E)-\int_{E} h_{n} d\left(\mu_{2}+i \nu_{2}\right)$

$$
\leqq\left|\alpha_{2}^{\prime \prime}(E)-\int_{E} k_{n} d \mu_{2}\right|+\left|\int_{E} k_{n} d \mu_{2}-\int_{E} h_{n}\left(1+i l_{n}\right) d \mu_{2}\right|
$$




$$
\begin{aligned}
& +\left|\int_{E} h_{n}\left(1+i l_{n}\right) d \mu_{2}-\int_{E} h_{n} d\left(\mu_{2}+i \nu_{2}\right)\right| \\
& <\frac{\varepsilon}{2}+0+\left|\int_{E} i h_{n} l_{n} d \mu_{2}-\int_{E} i h_{n} d \nu_{2}\right| \\
& \leqq \frac{\varepsilon}{2}+K_{n} \cdot \nu\left(\int l_{n} d \mu_{2}-\nu_{2}, E\right)<\frac{\varepsilon}{2}+K_{n} \cdot \frac{1}{n K_{n}} \\
& <\frac{\varepsilon}{2}+\frac{\varepsilon}{2}=\varepsilon \text { if } n \geqq N \text {. }
\end{aligned}
$$

Therefore

$$
\lim _{n} \int_{E} h_{n} d\left(\mu_{2}+i \nu_{2}\right)=\alpha_{2}^{\prime \prime}(E)
$$

uniformly for $E \in \Sigma$.

Noting that $\alpha_{2}^{\prime \prime} \perp \mu_{1}+i \nu_{1}$ and applying Lemma 1 again yields the existence of a sequence $\left\{h_{n}^{\prime}\right\}$ of $(S, \Sigma: C)$-simple functions such that

$$
\lim _{n} \int_{E} h_{n}^{\prime} d\left(\mu_{2}+i \nu_{2}+\left(\mu_{1}+i \nu_{1}\right)\right)=\lim _{n} \int_{E} h_{n}^{\prime} d \beta=\alpha_{2}^{\prime \prime}(E)
$$

uniformly for $E \in \Sigma$.

Considering (A), (B), and (C) together we have

$$
\lim _{n} \int_{E}\left(f_{n}^{\prime}+\left(-i g_{n}^{\prime}\right)+h_{n}^{\prime}\right) d \beta=\left(\alpha_{1}+\left(\alpha_{2}^{\prime}+\alpha_{2}^{\prime \prime}\right)\right)(E)=\alpha(E)
$$

uniformly for $E \in \Sigma$. Thus, let $z_{n}=\left(f_{n}^{\prime}+i\left(-g_{n}^{\prime}\right)+h_{n}^{\prime}\right)$ and the theorem is proved.

\section{REFERENCES}

1. S. Bochner, Additive set functions on groups, Ann. of Math. 40 (1939), 769-799.

2. S. Bochner and R. S. Phillips, Additive set functions and vector lattices, Ann. of Math. 42 (1941), 316-324.

3. R. B. Darst, $A$ decomposion of finitely additive set functions, J. Math. Reine Angew. 210 (1962), 31-37.

4. — A decomposition for complete normed abelian groups with applications to spaces of additive set functions, Trans. Amer. Math. Soc. 103 (1962), 549-559.

5. —_, The Lebesgue decomposition, Duke Math. J. 30 (1963), 553-556.

6. N. Dunford and J. T. Schwartz, Linear Operators, Interscience, New York, 1958.

7. C. Fefferman, $A$ Radon-Nikodym Theorem for finitely additive set functions, Pacific J. Math. 23 (1967), 35-45.

8. S. Leader, The theory of $L^{p}$-spaces for finitely additive set functions, Ann. of Math. 58 (1953), 528-543.

Received January 10, 1968. R. B. Darst was partially supported by a NSF grant, and Euline Green was partially supported by a NDEA Title IV fellowship. 



\section{PACIFIC JOURNAL OF MATHEMATICS}

\section{EDITORS}

\section{H. ROYDEN}

Stanford University

Stanford, California

\author{
R. R. Phelps \\ University of Washington \\ Seattle, Washington 98105
}

\section{J. DugundJI}

Department of Mathematics University of Southern California Los Angeles, California 90007

\section{RICHARD ARENS}

University of California

Los Angeles, California 90024

\section{ASSOCIATE EDITORS}

\section{E. F. BECKENBACH}

B. H. NEUMANN

F. WOLF

K. YOSIDA

\section{SUPPORTING INSTITUTIONS}

UNIVERSITY OF BRITISH COLUMBIA CALIFORNIA INSTITUTE OF TECHNOLOGY UNIVERSITY OF CALIFORNIA MONTANA STATE UNIVERSITY UNIVERSITY OF NEVADA NEW MEXICO STATE UNIVERSITY OREGON STATE UNIVERSITY UNIVERSITY OF OREGON OSAKA UNIVERSITY UNIVERSITY OF SOUTHERN CALIFORNIA

\author{
STANFORD UNIVERSITY \\ UNIVERSITY OF TOKYO \\ UNIVERSITY OF UTAH \\ WASHINGTON STATE UNIVERSITY \\ UNIVERSITY OF WASHINGTON \\ AMERICAN MATHEMATICAL SOCIETY \\ CHEVRON RESEARCH CORPORATION \\ TRW SYSTEMS
}

NAVAL WEAPONS CENTER

Mathematical papers intended for publication in the Pacific Journal of Mathematics should be in typed form or offset-reproduced, double spaced with large margins. Underline Greek letters in red, German in green, and script in blue. The first paragraph or two must be capable of being used separately as a synopsis of the entire paper. It should not contain references to the bibliography. Manuscripts, in duplicate if possible, may be sent to any one of the four editors. All other communications to the editors should be addressed to the managing editor, Richard Arens, University of California, Los Angeles, California 90024.

Each author of each article receives 50 reprints free of charge; additional copies may be obtained at cost in multiples of 50 .

The Pacific Journal of Mathematics is published monthly. Effective with Volume 16 the price per volume (3 numbers) is $\$ 8.00$; single issues, $\$ 3.00$. Special price for current issues to individual faculty members of supporting institutions and to individual members of the American Mathematical Society: $\$ 4.00$ per volume; single issues $\$ 1.50$. Back numbers are available.

Subscriptions, orders for back numbers, and changes of address should be sent to Pacific Journal of Mathematics, 103 Highland Boulevard, Berkeley 8, California.

Printed at Kokusai Bunken Insatsusha (International Academic Printing Co., Ltd.), 7-17, Fujimi 2-chome, Chiyoda-ku, Tokyo, Japan.

PUBLISHED BY PACIFIC JOURNAL OF MATHEMATICS, A NON-PROFIT CORPORATION

The Supporting Institutions listed above contribute to the cost of publication of this Journal, but they are not owners of publishers and have no responsibility for its content or policies. 


\section{Pacific Journal of Mathematics}

\section{Vol. 27, No. $2 \quad$ February, 1968}

Leonard E. Baum and George Roger Sell, Growth transformations for

functions on manifolds ............................ 211

Henry Gilbert Bray, A note on CLT groups ................... 229

Paul Robert Chernoff, Richard Anthony Rasala and William Charles

Waterhouse, The Stone-Weierstrass theorem for valuable fields....... 233

Douglas Napier Clark, On matrices associated with generalized

interpolation problems ................................

Richard Brian Darst and Euline Irwin Green, On a Radon-Nikodym theorem for finitely additive set functions . ...................... 255

Carl Louis DeVito, A note on Eberlein's theorem..................... 261

P. H. Doyle, III and John Gilbert Hocking, Proving that wild cells exist . . . 265

Leslie C. Glaser, Uncountably many almost polyhedral wild $(k-2)$-cells in

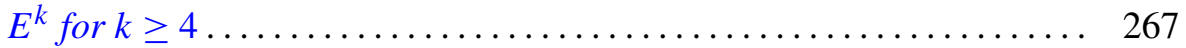

Samuel Irving Goldberg, Totally geodesic hypersurfaces of Kaehler

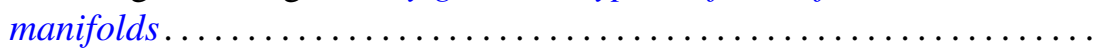

Donald Goldsmith, On the multiplicative properties of arithmetic functions .................................... 283

Jack D. Gray, Local analytic extensions of the resolvent ............ 305

Eugene Carlyle Johnsen, David Lewis Outcalt and Adil Mohamed Yaqub,

Commutativity theorems for nonassociative rings with a finite division ring homomorphic image ....................

André (Piotrowsky) De Korvin, Normal expectations in von Neumann

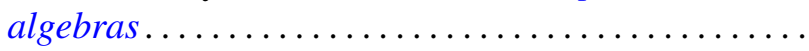

James Donald Kuelbs, A linear transformation theorem for analytic

Feynman integrals..........................

W. Kuich, Quasi-block-stochastic matrices ................... 353

Richard G. Levin, On commutative, nonpotent archimedean

semigroups ............................... 365

James R. McLaughlin, Functions represented by Rademacher series ... . . . 373

Calvin R. Putnam, Singular integrals and positive kernels............ 379

Harold G. Rutherford, II, Characterizing primes in some noncommutative

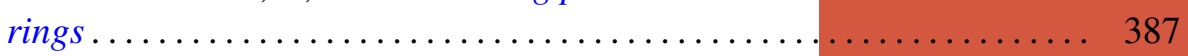

Benjamin L. Schwartz, On interchange graphs................... 393

Satish Shirali, On the Jordan structure of complex Banach *algebras . . . . . 397

Earl J. Taft, A counter-example to a fixed point conjecture............. 405

J. Roger Teller, On abelian pseudo lattice ordered groups ..... 